and choledocholithiasis. An important factor which produced such differences was reasonably assumed to be not only an organic but also functional disturbance in the papillary region.

III. In cases who had sphincteroplasty, the manometer was inserted into the terminal portion of the choledochus. Intrabiliary pressure were also measured in subjects who had no sphincteroplasty. The pressure of the choledochus after sphincteroplasty was $50-100 \mathrm{mmH}_{2} \mathrm{O}$, and the pressure of the pancreatic duct was $70 \mathrm{mmH}_{2} \mathrm{O}$, while the pressure without sphincteroplasty was $100 \mathrm{mmH}_{2} \mathrm{O}$.

\section{CONCLUSION}

In cases with choledocholithiasis, the lesion in the papillary region was noticed in extraordinary high rate in comparison with cholecystolithiasis. This lesion seemed to be one of the important factors that builds up the manifestation of cholelithiasis. The lesion was highly functional, though it was based on the organic changes. The significance of the lesion was emphasized.

\section{REFERENCES}

1) Kobayashi K, Mitani E, Yamada H: Studies on the functional disturbances of the papillary region using a pressure sensor. Gastroenterologia Japonica, 11 : 293-299, 1976.

2) Mitani E, Kobayashi K, Yamada H: The functional disorder of Oddi's muscle. Asian Medical Journal, 18, Stethoscope, 1975.

3) Kobayashi K, et al: Endoscopic observation in diseases of the biliary tract (mainly the so-called papillitis), Proceeding of the 1st Asian Pacific Congress of Endoscopy, 337, 1973.

\title{
(3) Structures and Developmental Mechanism of Gallstones
}

\author{
Fumitake Ishinara, M D and Hiroshi Nishikawa, M D
}

The First Department of Internal Medicine, The Jikei University School of Medicine, Tokyo

Studies on the mechanism of precipitation of bilirubin and cholesterol in the bile have made great progress recently. Two theories have been advocated on the pathogenesis of gallstones: development from the core, and development from the outer side to the center.

However, there is still great difficulty in explaining the formation of some of the gallstones. Metalic salts may be concerned in production of gallstone but their effects seem indefinite. In this paper, the morphological features and inorganic components of gallstones are reported.

\section{METHODS}

Structures of external and cut-surface of gallstones were observed by the stereoscopic microscope. The scanning electron microscope and polarizing microscope were applied to examine the morphological characteristics and development of crystals. The infrared ray spectrum analyzer and energy dipersive X-ray analyzer were used for detection, analysis and distribution of inorganic components.

\section{RESULTS}

The structures of gallstones can be classified as shown on Table 1 . In the radial type, two types are recognized: the radial type and the subradial one. In the former, wide crystals develop straightly toward the periphery. On the other hand, the latter has a core in the central region and crystals grow from the core to the external side. 
Table 1. Structural classification of gallstones
I Radial Type
radial $\ldots \ldots \ldots \ldots \ldots \ldots \ldots \ldots$ cases
subradial............. 7 cases
II Porous Type
without central open-space $\cdots 12$ cases
with central open-space $\cdots \cdots \cdot 11$ cases
III Poly-core Type
without enclusting layers ....4 4 cases
with enclusting layers $\ldots \ldots \ldots 1$ case
IV Concentric Type

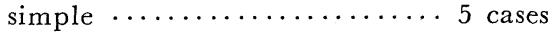
radial-concentric .......... 3 cases
subradial-concentric ....... 3 cases
V Massive Type ............. 2 cases
total 51 cases

The radial type is usually found in pure cholesterol stones and the subradial one is seen in mixed cholesterol stones.

In the porous type, two types are also observed. The type without central openspace has narrow crystals which extend to the outer side irregularly and at the same time further development is seen at the most external surface. The gallstones of this type have many characteristic tiny pores. In the type with central open-spaces, an open-space like a cavity is observed in the central region. The components in these types are mixed cholesterol.

In the poly-core type, two types are recognized. In the type without enclusting layers, many cores are seen to be aggregated, and the other type with enclusting layers has many layers around the cores.

The scanning electron microscopic findings in this type show that numerous granules in the core form a mulberry aggregation. The former is found in some mixed cholesterol, bilirubin, and black stones, and the latter contains bilirubin.

There are three types in the concentric type. The first is called the simple type which shows only a concentric circle. The second is the radial-concentric type in which radial structures in the central region and concentric structures in the external region are observed. The third is the subradialconcentric type which has subradial structures in the central region. The component in the simple type is bilirubin, and the other two types contain cholesterol.

Table 2. Structure and developmental mode of gallstones.

\begin{tabular}{|c|c|c|c|c|}
\hline & Classification & Cut-surface & $\begin{array}{l}\text { Polarizing microscopic } \\
\text { findings }\end{array}$ & Developmental mode \\
\hline I & radial type & & & \\
\hline II & porous type & 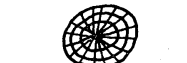 & 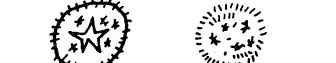 & \\
\hline III & poly-core type & & (0) & \\
\hline \multirow{2}{*}{ IV } & concentric type & & & \\
\hline & $\begin{array}{l}\text { radial-concentric } \\
\text { type }\end{array}$ & & 1 & \\
\hline V & massive type & & & \\
\hline
\end{tabular}


The massive type is indefinite and also has no morphological features. Some of the black stones can be seen in this type.

As to inorganic components, Ca, S, Cl, $\mathrm{P}$, and $\mathrm{Si}$ are observed and regardless of the kinds of gallstones, Ca elements are the richest in all of them. Their distribution tends to be localized in the center. Bilirubin stones have more inorganic components than cholesterol stones. Each element is localized between crystals.

\section{DISCUSSION}

The developmental mode of gallstone from observations on their morphological features are illustrated in Table 2. Gallstones of the radial type develop directly from the center, but in the subradial type the core is formed at first and then radial development takes place.

These findings suggest the complicated growth formation.

The production route of the porous type is regarded that after formation of the external surface, condensing changes may occur during development to the center, so both outer and inner formation can be supposed. Many changes such as dissolution or recrystallization may take place in this type.

In the poly-core type, many granules are aggregated in the core and this suggests that gallstones of this type develop toward the outer side.
In the concentric type, growth layers are found and it shows that this type is producted from the inner to the outer side.

The massive type may develop to the external side, but because of having no typical features, its formation is obscure.

Many kinds of inorganic components are seen in gallstones and a large quantity of $\mathrm{Ca}$ elements are observed in the central region.

Their distributions are localized specifically between crystals. These facts indicate that inorganic components may have an important influence in unstablization and coagulation of bile colloid and in formation of gallstone.

\section{SUMMARY}

Morphological classification and developmental mode of gallstones have been demonstrated.

The radial type grows toward the external side. In the porous type, both outer and inner development can be supposed and a complicated mechanism must be taken into consideration.

The poly-core, concentric and radialconcentric types develop from the center to the outer side and the massive type may be producted from the center.

A large quantity of $\mathrm{Ca}$ elements are contained in gallstone and they show a specific distribution and are presumed to take part in gallstone formation.

\title{
(4) Pathogenesis of Cholesterol Gallstone
}

\author{
Toshiaki Osuga, M.D. \\ Department of Internal Medicine, The University of Tsukuba School of Medicine, \\ Sakura-mura, Niiharr-gun, Ibaraki Pref.
}

The mechanism of cholesterol gallstone formation is discussed comparing human and alimentarily induced cholelithiasis in squirrel monkeys.
The process of cholesterol gallstone formation can be divided to three phases. 1) host reaction due to predisposition and risk factors 2) production of bile supersaturated 